\title{
Electroweak phase transition in an inert complex triplet model
}

\author{
MJ. Kazemi* and S. S. AbdusSalam $\oplus^{\dagger}$ \\ Department of Physics, Shahid Beheshti University, Tehran 19839, Islamic Republic of Iran
}

(Received 6 November 2020; accepted 28 February 2021; published 12 April 2021)

\begin{abstract}
We study the dynamics of the electroweak phase transition in a simple extension of the Standard Model where the Higgs sector is extended by adding an $S U(2)_{L}$ triplet with hypercharge $Y=2$. By making random scans over the parameters of the model, we show that there are regions consistent with constraints from collider experiments and the requirement for a strong first-order electroweak phase transition which is needed for electroweak baryogenesis. Further, we also study the power spectrum of the gravitational waves that can be generated due to the first-order phase transitions. Moreover, the detectability of these gravitational waves, via future space-based detectors, is discussed.
\end{abstract}

DOI: 10.1103/PhysRevD.103.075012

\section{INTRODUCTION}

A cosmological electroweak phase transition (EWPT) is interesting for numerous reasons. It can be a source of primordial magnetic fields [1], generate detectable background gravitational waves [2], affect the abundance of thermal relic densities for candidate dark matter particles [3], and, perhaps most importantly, lead to suitable preconditions for baryogenesis [4].

The Planck Collaboration [5] measured the baryon-tophoton ratio of the Universe as

$$
\frac{n_{b}}{n_{\gamma}}=(6.10 \pm 0.04) \times 10^{-10}
$$

This is consistent with astronomical measurements of lightelement abundances, assuming standard big bang nucleosynthesis $[4,6]$. This measured value represents one of the big unresolved particle physics puzzles. It quantifies the matter-antimatter asymmetry of the Universe $[4,7]$. The mechanism(s) behind the asymmetry needs to be determined and understood. About half a century ago, Sakharov proposed three early Universe conditions that must be satisfied for successful baryon asymmetry generation [8]: i) baryon number violation, ii) $C$ and $C P$ violation, and iii) a departure from thermal equilibrium. These are in principle possible within the framework of an electroweak phase transition in the early Universe called electroweak baryogenesis (EWBG). The realization of EWBG within the

\footnotetext{
*mj_kazemi@sbu.ac.ir

abdussalam@sbu.ac.ir
}

Published by the American Physical Society under the terms of the Creative Commons Attribution 4.0 International license. Further distribution of this work must maintain attribution to the author(s) and the published article's title, journal citation, and DOI. Funded by SCOAP.
Standard Model (SM) of particle physics turned out to be problematic according to lattice simulations [9]. It was found that only crossover (instead of strongly first-order) phase transitions are possible in the early Universe for the observed value of the Higgs boson mass. This indicates that some physics beyond the SM is essential.

One of the simplest classes of models beyond the SM that may lead to strong first-order phase transitions and successful EWBG can be made by adding an electroweak scalar $S U(2)_{L}$ multiplet to the SM Higgs sector. A global $Z_{2}$ symmetry is imposed when constructing these so-called inert multiplet models. With the $Z_{2}$ symmetry, the lightest neutral component of the new scalar multiplet can be considered as a candidate for dark matter [10,11].

The EWPT and its gravitational-wave signatures have been well studied within the framework of the singlet [1215], doublet [16,17], and real triplet $(Y=1)$ [17-19] cases of the scalar-multiplet class of models. Another wellmotivated representation of the $S U(2)_{L}$ group is the complex triplet $(Y=2)$, which could also be used to explain the smallness of the neutrino mass in the type II seesaw mechanism [20-22]. In this article we address the EWPT of the inert complex triplet model by scanning its parameter space with experimental constraints imposed from Higgs signal strengths. We analyze the parameterspace regions that could lead to a first-order EWPT. We also study the power spectra of gravitational waves that could be generated following the first-order transitions. The observation of such gravitational waves, such as by future space-based gravitational-wave detectors [23], could yield information that is complementary to collider and dark matter experiments.

This paper is organized as follows. In Sec. II we briefly review the inert complex triplet model, and describe the relevant parameters for the EWPT and the theoretical constraints taken into account. In Sec. III we describe 
the results from collider experiments regarding Higgs decay to diphotons and use these to constrain the parameter space of the inert complex triplet model. Finally, in Secs. IV and V we present numerical analyses of the model in light of the EWPT and gravitational-wave generation, respectively.

\section{THE MODEL}

We extend the SM Higgs sector by adding one complex scalar $S U_{L}(2)$ triplet $\Delta$ with hypercharge $Y=2$,

$$
\Delta=\left(\begin{array}{c}
\Delta^{++} \\
\Delta^{+} \\
\Delta^{0} \equiv \frac{1}{\sqrt{2}}(S+i A)
\end{array}\right),
$$

and impose a $Z_{2}$ discrete symmetry, under which $\Delta \rightarrow-\Delta$ and all other fields are unchanged. The most general scalar potential, symmetric under $Z_{2}$, involving this triplet and the standard $S U_{L}(2)$ Higgs doublet

$$
H=\left(\begin{array}{c}
G^{+} \\
\frac{1}{\sqrt{2}}\left(\phi+i G^{0}\right)
\end{array}\right)
$$

can be written in the following form [11,24]:

$$
\begin{aligned}
V_{0}= & -\mu_{H}^{2} H^{\dagger} H+\lambda_{H}\left(H^{\dagger} H\right)^{2} \\
& +\mu_{\Delta}^{2} \Delta^{\dagger} \Delta+\lambda_{\Delta}^{(1)}\left(\Delta^{\dagger} \Delta\right)^{2}+\lambda_{\Delta}^{(2)}\left|\Delta^{\dagger} T^{a} \Delta\right|^{2} \\
& +\lambda_{H \Delta}^{(1)} H^{\dagger} H \Delta^{\dagger} \Delta+\lambda_{H \Delta}^{(2)} H^{\dagger} \tau^{a} H \Delta^{\dagger} T^{a} \Delta .
\end{aligned}
$$

Here, $\tau^{a}$ and $T^{a}$ are the $S U(2)$ generators in the fundamental and 3 representations, respectively. These are normalized such that $\operatorname{Tr}\left[\tau^{a}, \tau^{b}\right]=\frac{1}{2} \delta^{a b}$ and $\operatorname{Tr}\left(T^{a} T^{b}\right)=\frac{1}{2} \delta^{a b}$. Explicitly, $\tau^{a}=\frac{1}{2} \sigma^{a}$, where $\sigma^{a}$ are Pauli matrices and $T^{a}$ are

$$
\begin{aligned}
T^{1} & =\frac{1}{\sqrt{2}}\left(\begin{array}{ccc}
0 & -1 & 0 \\
-1 & 0 & 1 \\
0 & 1 & 0
\end{array}\right), \quad T^{2}=\frac{1}{\sqrt{2}}\left(\begin{array}{ccc}
0 & i & 0 \\
-i & 0 & -i \\
0 & i & 0
\end{array}\right), \\
T^{3} & =\left(\begin{array}{ccc}
1 & 0 & 0 \\
0 & 0 & 0 \\
0 & 0 & -1
\end{array}\right) .
\end{aligned}
$$

We require that $\Delta$ be odd under $Z_{2}$ symmetry so that the neutral component will not acquire a vacuum expectation value. There is an electroweak-symmetry-breaking minimum at zero temperature, with $\left\langle H^{T}\right\rangle=(0, v / \sqrt{2})$ and $\left\langle\Delta^{T}\right\rangle=(0,0,0)$. In this case, the tree-level field-dependent masses of Standard Model particles are the same as in the SM,

$$
\begin{aligned}
m_{t}^{2}(\phi) & =\frac{y_{t}^{2}}{2} \phi^{2}, \quad m_{b}^{2}(\phi)=\frac{y_{b}^{2}}{2} \phi^{2}, \\
m_{W}^{2}(\phi) & =\frac{g^{2}}{4} \phi^{2}, \quad m_{Z}^{2}(\phi)=\frac{g^{2}+g^{2}}{4} \phi^{2}, \\
m_{h}^{2}(\phi) & =-\mu_{H}^{2}+3 \lambda_{H} \phi^{2},
\end{aligned}
$$

and the masses of the components of the additional triplet scalar are given by

$$
\begin{aligned}
m_{S}^{2}(\phi) & =m_{A}^{2}(\phi)=\mu_{\Delta}^{2}+\frac{1}{2}\left(\lambda_{H \Delta}^{(1)}+\frac{1}{2} \lambda_{H \Delta}^{(2)}\right) \phi^{2}, \\
m_{\Delta^{+}}^{2}(\phi) & =\mu_{\Delta}^{2}+\frac{1}{2} \lambda_{H \Delta}^{(1)} \phi^{2}, \\
m_{\Delta^{++}}^{2}(\phi) & =\mu_{\Delta}^{2}+\frac{1}{2}\left(\lambda_{H \Delta}^{(1)}-\frac{1}{2} \lambda_{H \Delta}^{(2)}\right) \phi^{2} .
\end{aligned}
$$

This model has five real parameters in addition to those of the SM. However, only three of them, i.e., the triplet mass parameter and the doublet-triplet couplings, appear in the tree-level triplet masses. Thus, only $\mu_{\Delta}, \lambda_{H \Delta}^{(1)}$, and $\lambda_{H \Delta}^{(2)}$ are relevant for electroweak phase transition dynamics in the one-loop approximation (see Sec. III).

In the next section, we first study some constraints on these parameters that come from collider phenomenology (or, equivalently, on the mass spectrum of the triplet scalar at $\langle\phi\rangle=v=246 \mathrm{GeV}$ ). After this, we study the parameter space to find regions that can lead to (i) strong first-order phase transitions and (ii) detectable gravitational waves. While sampling the parameter space, we apply theoretical constraints, checking that unitarity and vacuum stability conditions on the triplet self-couplings are satisfied before applying those from the Higgs signal strength measurements. For a stable vacuum, the scalar potential should be bounded from below along all possible field directions. At the tree level, the vacuum stability requirement leads to [11]

$$
\begin{aligned}
& \lambda_{H}, \lambda_{\Delta}^{(1)}, \lambda_{\Delta}^{(2)}>0-2 \sqrt{\lambda_{H}\left(\lambda_{\Delta}^{(1)}+\lambda_{\Delta}^{(2)}\right)}<2 \lambda_{H \Delta}^{(1)}+\lambda_{H \Delta}^{(2)} \\
& -2 \sqrt{\lambda_{H} \lambda_{\Delta}^{(1)}}<\lambda_{H \Delta}^{(1)}-2 \sqrt{\lambda_{H}\left(\lambda_{\Delta}^{(1)}+\lambda_{\Delta}^{(2)}\right)}<2 \lambda_{H \Delta}^{(1)}-\lambda_{H \Delta}^{(2)} .
\end{aligned}
$$

In what follows, we begin by considering the implications of $h \rightarrow \gamma \gamma$ and $h \rightarrow Z \gamma$ limits on the parameter regions of the inert complex triplet model, which from now on we call the inert triplet model (ITM).

\section{EXCLUDING PARAMETER SPACE VIA HIGGS DECAY RATES}

The branching ratios of the Higgs decays in the ITM differ from the SM ones. As such, the Higgs decay measurement or limits can be used as a probe for the ITM. Specifically, the Higgs-to-diphoton channel, $h \rightarrow \gamma \gamma$, because of its relatively clean signature at the Large Hadron 
Collider (LHC), could play an important role for this purpose. Here we analyze the ITM parameter space by using recent ATLAS and CMS results for the Higgs-todiphoton signal strength. We find that a significantly large region of the parameter space is excluded via these recent data.

To study the ITM contributions to the $h \rightarrow \gamma \gamma$ decay rate, we address the ratio

$$
\begin{aligned}
R_{\gamma \gamma} & \equiv \frac{\sigma(p p \rightarrow h \rightarrow \gamma \gamma)^{\mathrm{ITM}}}{\sigma(p p \rightarrow h \rightarrow \gamma \gamma)^{\mathrm{SM}}} \\
& \approx \frac{[\sigma(g g \rightarrow h) \operatorname{Br}(h \rightarrow \gamma \gamma)]^{\mathrm{TTM}}}{[\sigma(g g \rightarrow h) \operatorname{Br}(h \rightarrow \gamma \gamma)]^{\mathrm{SM}}} .
\end{aligned}
$$

Here, we used the fact that gluon-gluon fusion is the dominant channel for Higgs production. Moreover, since $\sigma(g g \rightarrow h)$ is the same in both the ITM and SM, the $R_{\gamma \gamma}$ reduces to [25]

$$
R_{\gamma \gamma}=\frac{\Gamma_{h}^{\mathrm{SM}}}{\Gamma_{h}^{\mathrm{TTM}}} \frac{\Gamma(h \rightarrow \gamma \gamma)^{\mathrm{ITM}}}{\Gamma(h \rightarrow \gamma \gamma)^{\mathrm{SM}}} .
$$

In a similar way, for the $Z \gamma$ decay channel an analogous quantity, $R_{Z \gamma}$, can be defined as

$$
R_{Z \gamma}=\frac{\Gamma_{h}^{\mathrm{SM}}}{\Gamma_{h}^{\mathrm{ITM}}} \frac{\Gamma(h \rightarrow Z \gamma)^{\mathrm{ITM}}}{\Gamma(h \rightarrow Z \gamma)^{\mathrm{SM}}} .
$$

Within the SM, many channels contribute to the total decay width of the Higgs boson. The most important ones for $m_{h}=125 \mathrm{GeV}$ are $b \bar{b}, c \bar{c}, \tau^{+} \tau^{-}, Z Z^{*}, W W^{*}, \gamma \gamma, Z \gamma$, and $g g$. Hence, the total Higgs decay width is approximately given by

$$
\begin{aligned}
\Gamma_{h}^{\mathrm{SM}}= & \sum_{f=\tau, b, c} \Gamma_{h \rightarrow f \bar{f}}^{\mathrm{SM}}+\Gamma_{h \rightarrow W W^{*}}^{\mathrm{SM}} \\
& +\Gamma_{h \rightarrow Z Z^{*}}^{\mathrm{SM}}+\Gamma_{h \rightarrow g g}^{\mathrm{SM}}+\Gamma_{h \rightarrow \gamma \gamma}^{\mathrm{SM}}+\Gamma_{h \rightarrow Z \gamma}^{\mathrm{SM}} .
\end{aligned}
$$

In the ITM, the total decay width of the Higgs can be modified with respect to the SM, since the charged scalars exchanged in loops give extra contributions to the $h \rightarrow \gamma \gamma$ and $h \rightarrow Z \gamma$ amplitudes (see Fig. 1) [26]. In addition, the total decay width changes due to the existence of additional decay channels, i.e., $h \rightarrow S S, h \rightarrow A A, h \rightarrow \Delta^{ \pm} \Delta^{\mp}$, and $h \rightarrow \Delta^{ \pm \pm} \Delta^{\mp \mp}[25,27]$. So,

$$
\begin{aligned}
\Gamma_{h}^{\mathrm{ITM}}= & \sum_{f=\tau, b, c} \Gamma_{h \rightarrow f \bar{f}}^{\mathrm{ITM}}+\Gamma_{h \rightarrow W W^{*}}^{\mathrm{ITM}} \\
& +\Gamma_{h \rightarrow Z Z^{*}}^{\mathrm{ITM}}+\Gamma_{h \rightarrow g g}^{\mathrm{ITM}}+\Gamma_{h \rightarrow \gamma \gamma}^{\mathrm{ITM}}+\Gamma_{h \rightarrow Z \gamma}^{\mathrm{ITM}} \\
& +\Gamma_{h \rightarrow A A}^{\mathrm{ITM}}+\Gamma_{h \rightarrow S S}^{\mathrm{ITM}}+\Gamma_{h \rightarrow \Delta^{+} \Delta^{-}}^{\mathrm{ITM}}+\Gamma_{h \rightarrow \Delta^{++} \Delta^{--}}^{\mathrm{ITM}}
\end{aligned}
$$

The decay rates of these additional decay channels, when they are kinematically open $\left(2 m_{\varphi}<m_{h}\right)$ are given by

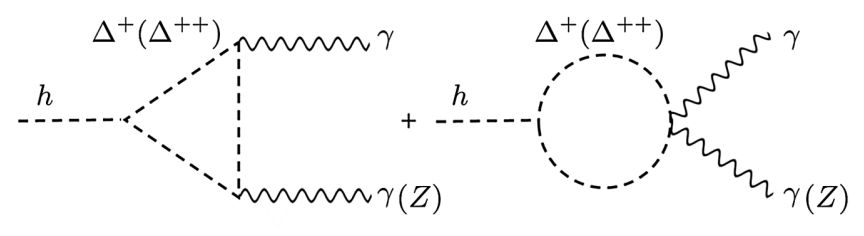

FIG. 1. Feynman diagrams for charged scalar particles contributing to $h \rightarrow \gamma \gamma$ or $h \rightarrow Z \gamma$.

$$
\Gamma_{h \rightarrow \varphi \varphi^{\dagger}}^{\mathrm{ITM}}=\frac{\xi_{\varphi} \lambda_{h \varphi \varphi^{\dagger}}^{2}}{8 \pi m_{h}} \sqrt{1-\frac{4 m_{\varphi}^{2}}{m_{h}^{2}}},
$$

where $\lambda_{h \varphi \varphi^{\dagger}}=\left(m_{\varphi}^{2}-\mu_{\Delta}^{2}\right) / v$ and $\xi_{\varphi}=1$ for $\varphi=A, S$ and $\lambda_{h \varphi \varphi^{\dagger}}=2\left(m_{\varphi}^{2}-\mu_{\Delta}^{2}\right) / v$ and $\xi_{\varphi}=1 / 2$ for charged scalars, $\varphi=\Delta^{+}, \Delta^{++}$.

The partial widths of the tree-level Higgs decays into SM particles and the loop-mediated decay into $g g$ in the ITM are equal to the corresponding ones in the SM; for completeness, they are summarized in Appendix A. The $h \rightarrow Z \gamma$ and $h \rightarrow \gamma \gamma$ SM processes are modified within the ITM. In the SM these decays are dominated by contributions from the $W$ gauge boson and top-quark loops, while in the ITM the couplings of the Higgs doublet to the triplet scalars modify these decays via the following one-loop diagrams.

Following the general results for spin- 0 , spin- $1 / 2$, and spin- 1 contributions to these decay rates $[26,28]$, which can be obtained using the Feynman rules listed in Ref. [29], the modified decays in the ITM are given by [30]

$$
\begin{gathered}
\Gamma_{H \rightarrow \gamma \gamma}^{\mathrm{ITM}}=\frac{\alpha^{2}}{256 \pi^{3} v^{2}} m_{h}^{3}\left|\mathcal{A}_{\mathrm{ITM}}^{\gamma \gamma}\right|^{2}, \\
\Gamma_{h \rightarrow Z \gamma}^{\mathrm{ITM}}=\frac{\alpha^{2}}{512 \pi^{3} v^{2}} m_{h}^{3}\left(1-\frac{m_{Z}^{2}}{m_{h}^{2}}\right)^{3}\left|\mathcal{A}_{\mathrm{ITM}}^{Z \gamma}\right|^{2} .
\end{gathered}
$$

Here,

$$
\begin{aligned}
& \mathcal{A}_{\mathrm{SM}}^{\gamma \gamma}=A_{1}^{\gamma \gamma}\left(\tau_{w}\right)+\frac{4}{3} A_{\frac{1}{2}}^{\gamma \gamma}\left(\tau_{t}\right), \\
& \mathcal{A}_{\mathrm{ITM}}^{\gamma \gamma}=\mathcal{A}_{\mathrm{SM}}^{\gamma \gamma}+g_{\Delta^{+}}^{\gamma \gamma} A_{0}^{\gamma \gamma}\left(\tau_{\Delta^{+}}\right)+g_{\Delta^{+}}^{\gamma \gamma} A_{0}^{\gamma \gamma}\left(\tau_{\Delta^{++}}\right), \\
& \mathcal{A}_{\mathrm{SM}}^{Z \gamma}=2 \frac{c_{w}}{s_{w}} A_{1}^{Z \gamma}\left(\tau_{W}, \lambda_{W}\right)+4 \frac{\left(1-\frac{8}{3} s_{w}^{2}\right)}{s_{w} c_{w}} A_{1 / 2}^{Z \gamma}\left(\tau_{t}, \lambda_{t}\right), \\
& \mathcal{A}_{\mathrm{ITM}}^{Z \gamma}=\mathcal{A}_{\mathrm{SM}}^{Z \gamma}-g_{\Delta^{+}}^{Z \gamma} A_{0}^{Z \gamma}\left(\tau_{\Delta^{+}}, \lambda_{\Delta^{+}}\right)-g_{\Delta^{++}}^{Z \gamma} A_{0}^{Z \gamma}\left(\tau_{\Delta^{++}}, \lambda_{\Delta^{++}}\right), \\
& \tau_{i}=4 m_{i}^{2} / m_{h}^{2}, \lambda_{i}=4 m_{i}^{2} / m_{Z}^{2}\left(i=W, t, \Delta^{+}, \Delta^{++}\right), s_{w}=\sin \theta_{w}, \\
& \text { and } c_{w}=\cos \theta_{w} \cdot \theta_{w} \text { is the Weinberg mixing angle and the } \\
& \text { coupling constants are given by }
\end{aligned}
$$




$$
\begin{aligned}
g_{\Delta^{+}}^{\gamma \gamma} & =\frac{m_{\Delta^{+}}^{2}-\mu_{\Delta}^{2}}{m_{\Delta^{+}}^{2}}, \\
g_{\Delta^{++}}^{\gamma \gamma} & =\frac{4\left(m_{\Delta^{++}}^{2}-\mu_{\Delta}^{2}\right)}{m_{\Delta^{++}}^{2}}, \\
g_{\Delta^{+}}^{Z \gamma} & =\frac{4\left(m_{\Delta^{+}}^{2}-\mu_{\Delta}^{2}\right)\left(-s_{w}^{2}\right)}{m_{\Delta^{+}}^{2} s_{w} c_{w}}, \\
g_{\Delta^{++}}^{Z \gamma} & =\frac{8\left(m_{\Delta^{++}}^{2}-\mu_{\Delta}^{2}\right)\left(1-2 s_{w}^{2}\right)}{m_{\Delta^{++}}^{2} s_{w} c_{w}} .
\end{aligned}
$$

The loop functions $A_{(0,1 / 2,1)}^{\gamma \gamma}$ and $A_{(0,1 / 2,1)}^{Z \gamma}$ are given in Appendix B.

In Fig. 2 we plot the branching ratios for some regions of parameter space. It turns out that when the triplet decay channels $\left(h \rightarrow A A, \quad h \rightarrow S S, \quad h \rightarrow \Delta^{+} \Delta^{-}\right.$, and $h \rightarrow \Delta^{++} \Delta^{--}$) are kinematically allowed, their partial widths dominate over the partial widths of decays into SM particles. Therefore, in this case the value of $R_{\gamma \gamma}$ deviates significantly from $R_{\gamma \gamma}=1$. This is not consistent with experimental results. Conversely, when these decay channels are kinematically closed $\left(m_{A}, m_{S}, m_{\Delta^{+}}, m_{\Delta^{++}}>m_{h} / 2\right)$, the total width of $h$ is slightly modified with respect to the SM case, since the branching ratios of $h \rightarrow \gamma \gamma$ and $h \rightarrow Z \gamma$, which are the only processes that receive contributions from triplet scalars, are of the order of $10^{-2}$.

For the numerical analysis, we scan the parameter space of the ITM in the range $10 \mathrm{GeV} \leq m_{S}, m_{\Delta^{++}} \leq 500 \mathrm{GeV}$, for some specific values of $\mu_{\Delta} ; 100,150,200$, and $250 \mathrm{GeV}$. We then compare the values of $R_{\gamma \gamma}$ obtained with the most recent measurements by the ATLAS [31] and CMS [32] collaborations:

$$
R_{\gamma \gamma}^{\text {ATLAS }}=0.99 \pm 0.14 \quad \text { and } \quad R_{\gamma \gamma}^{\text {CMS }}=1.18_{-0.14}^{+0.17} .
$$

In fact, we find that the $R_{\gamma \gamma}$ enhancement is only possible when $m_{S}, m_{\Delta^{++}}>m_{h} / 2$. In Fig. 3 we illustrate the regions of parameter space allowed by these experimental constraints. The colored bands represented the regions within the reported experimental uncertainties. We also superimpose contour lines that represent the values of $R_{Z \gamma}$. The decay $h \rightarrow Z \gamma$ has not been discovered [33], but $R_{Z \gamma}$ is bounded from above to be less than 3.6 at $95 \%$ C.L. [34].

In the next section we discuss how these experimental results can constrain the properties of electroweak phase transitions and the gravitational-wave spectra that could follow.

\section{DYNAMICS OF THE EWPT}

In order to study the electroweak phase transition, we need to follow the evolution of the Higgs vacuum expectation value, i.e., the minimum of the Higgs effective potential over the thermal history of the Universe. For this, we use the standard techniques of finite-temperature field theory $[35,36]$. The one-loop-level Higgs effective potential at finite temperature can be written as

$$
V(\phi, T)=V_{0}(\phi)+V_{\mathrm{CW}}(\phi)+V_{T}(\phi, T),
$$

where the tree-level potential is given by

$$
V_{0}(\phi)=-\frac{1}{2} \mu_{H} \phi^{2}+\frac{1}{4} \lambda_{H} \phi^{4} .
$$

The zero- and finite-temperature corrections at one loop, i.e., the Coleman-Weinberg potential $V_{\mathrm{CW}}(\phi)[37,38]$ and $V_{T}(\phi, T)$ are, respectively, given by $[39,40]$

$$
V_{\mathrm{CW}}=\sum_{i} g_{i}\left[m_{i}^{4}(\phi)\left(\log \frac{m_{i}^{2}(\phi)}{m_{i}^{2}\left(v_{0}\right)}-\frac{3}{2}\right)+2 m_{i}^{2}(\phi) m_{i}^{2}\left(v_{0}\right)\right]
$$

and
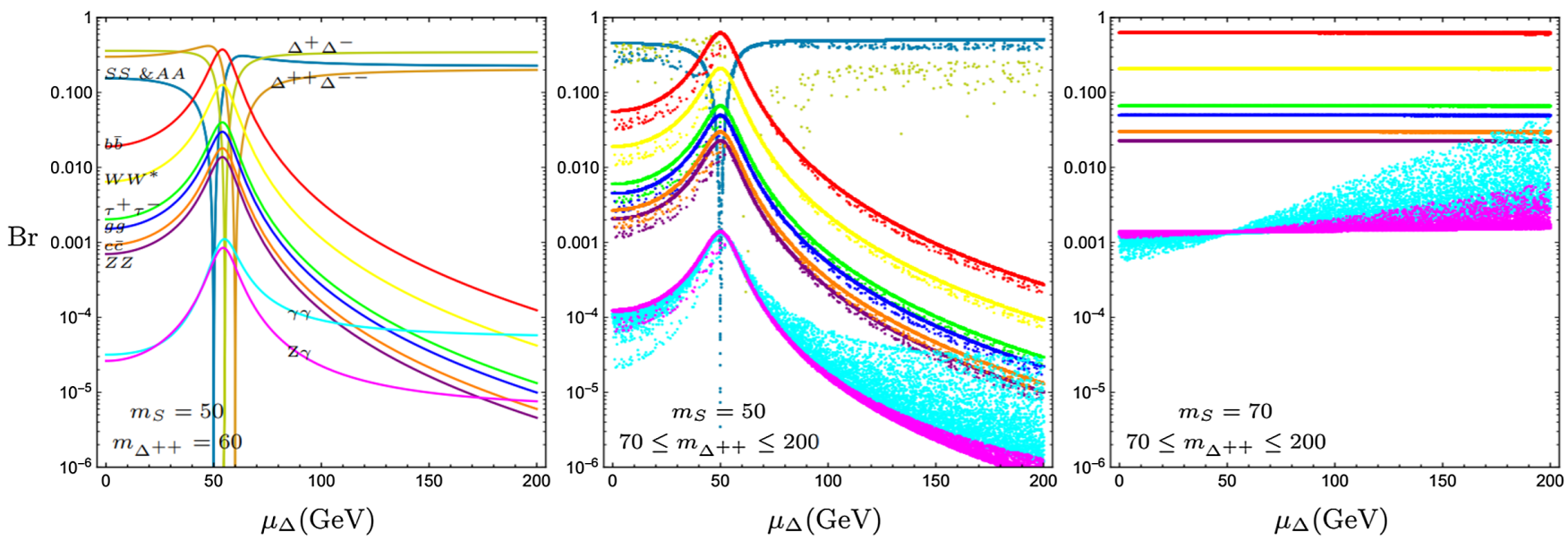

FIG. 2. Branching ratios for $h$ with mass $125 \mathrm{GeV}$. Left panel: decay channels $h \rightarrow A A, h \rightarrow S S, h \rightarrow \Delta^{+} \Delta^{-}$, and $h \rightarrow \Delta^{++} \Delta^{--}$are open $\left(m_{S}=m_{A}=50 \mathrm{GeV}, m_{\Delta^{++}}=60 \mathrm{GeV}\right)$. Middle panel: $h \rightarrow \Delta^{+} \Delta^{-}$and $h \rightarrow \Delta^{++} \Delta^{--}$are open. Right panel: no $h$ decay channels to triplet particles are allowed $\left(m_{S}=70 \mathrm{GeV}, m_{\Delta^{++}}>m_{\Delta^{+}}>70 \mathrm{GeV}\right)$. 

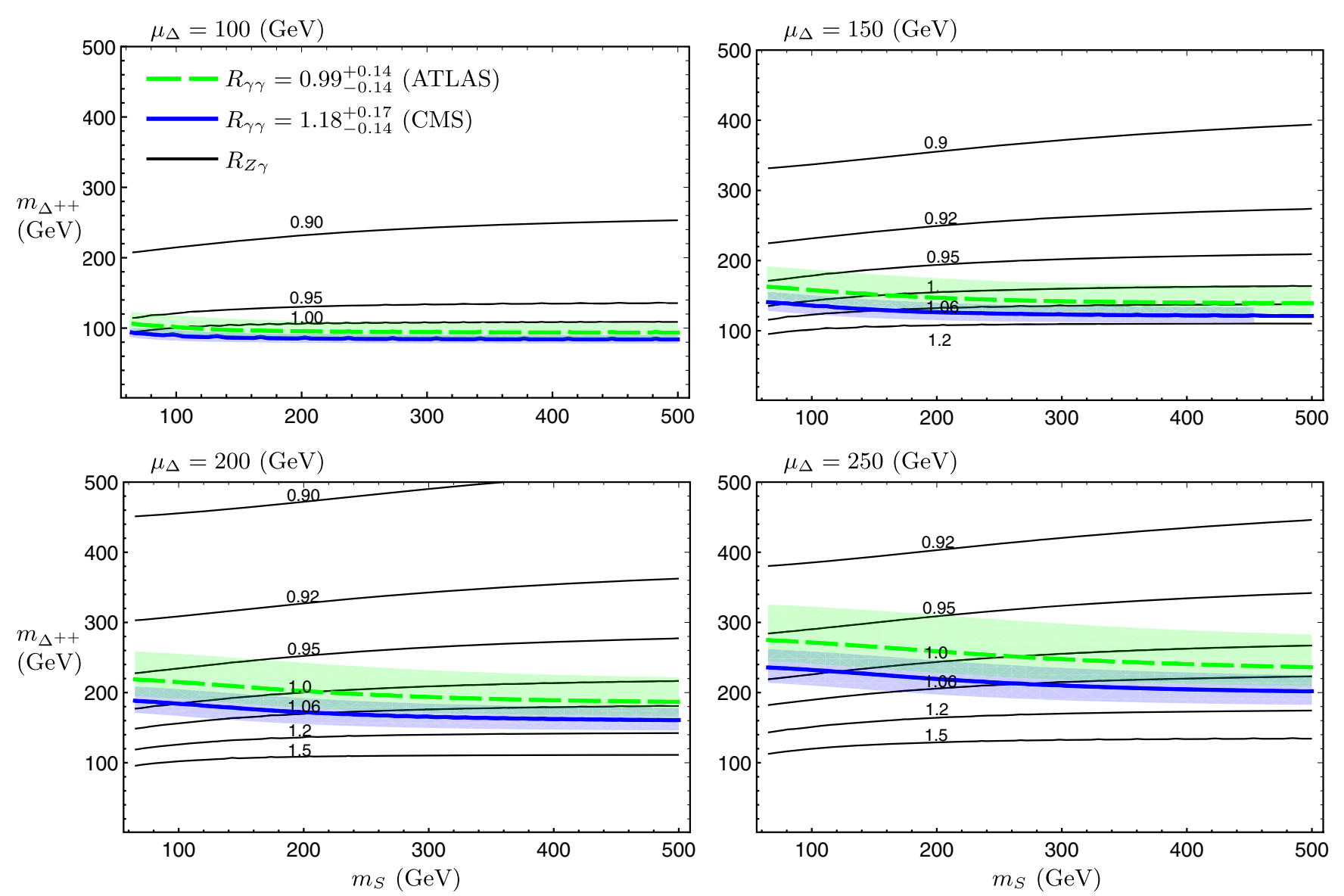

FIG. 3. Regions of the triplet mass spectrum that are consistent with the measured values of $R_{\gamma \gamma}$ by ATLAS and CMS, for $\mu_{\Delta}$ equal to 100, 150, 200, and $250 \mathrm{GeV}$. The solid black contour lines represent $R_{Z \gamma}$. The blue and green bands capture the $1 \sigma$ uncertainties for the ATLAS and CMS results. The light blue band is for the CMS observed value of $R_{\gamma \gamma}$ whose central value is represented by the solid blue line. The light green band is for the ATLAS observed value of $R_{\gamma \gamma}$ whose central value is represented by the broken green line.

$$
V_{T}=\sum_{i} 32 g_{i} T \int_{0}^{\infty} d x x^{2} \ln \left[1-(-1)^{F_{i}} e^{-\sqrt{x^{2}+m_{i}^{2}(\phi)} / T}\right]
$$

Here $i=\left\{W, Z, t, b, h, A, S, \Delta^{+}, \Delta^{++}\right\}, F_{i}$ represents the fermionic number, $n_{i}$ is the number of degrees of freedom of the different species of particles,

$$
n_{i}=\{6,3,12,12,1,1,1,2,2\}
$$

and $g_{i} \equiv(-1)^{F_{i}} n_{i} / 64 \pi^{2}$.

A first-order phase transition happens when the effective potential has two minima of the same value at some critical temperature $T_{c}$. In such a case, the system can transit between the vacua via thermal fluctuations or quantum tunneling. This transition physically signifies the creation of spherically symmetric regions of true vacuum, bubbles of the broken phase, expanding in the background of the false vacuum.

In the standard EWBG scenario, the SM fermions interact with the bubble walls in a $C P$-violating manner. This leads to a chiral asymmetry production in front of the bubble wall, which can subsequently give rise to baryon generation via sphaleron processes which convert the chiral asymmetry to baryon asymmetry $[4,41]$. The generated baryons could then fall into the growing bubble. This EWBG mechanism could explain the matter-antimatter asymmetry of the Universe if the generated baryon asymmetry is not washed out by sphalerons inside the bubble $[4,41]$. This condition requires that $[4,42]$

$$
\frac{\phi_{c}}{T_{c}}>1
$$

where $\phi_{c}$ is the Higgs vacuum expectation value at the critical temperature $T_{c}$. We numerically check this condition within the ITM by randomly scanning the ITM parameters allowed within the ranges

$$
10 \mathrm{GeV} \leq m_{S}, \quad m_{\Delta^{++}} \leq 500 \mathrm{GeV},
$$

for fixed values of $\mu_{\Delta}=100,150,200$, and $250 \mathrm{GeV}$. The results are shown in Fig. 4. It can be seen that there are 

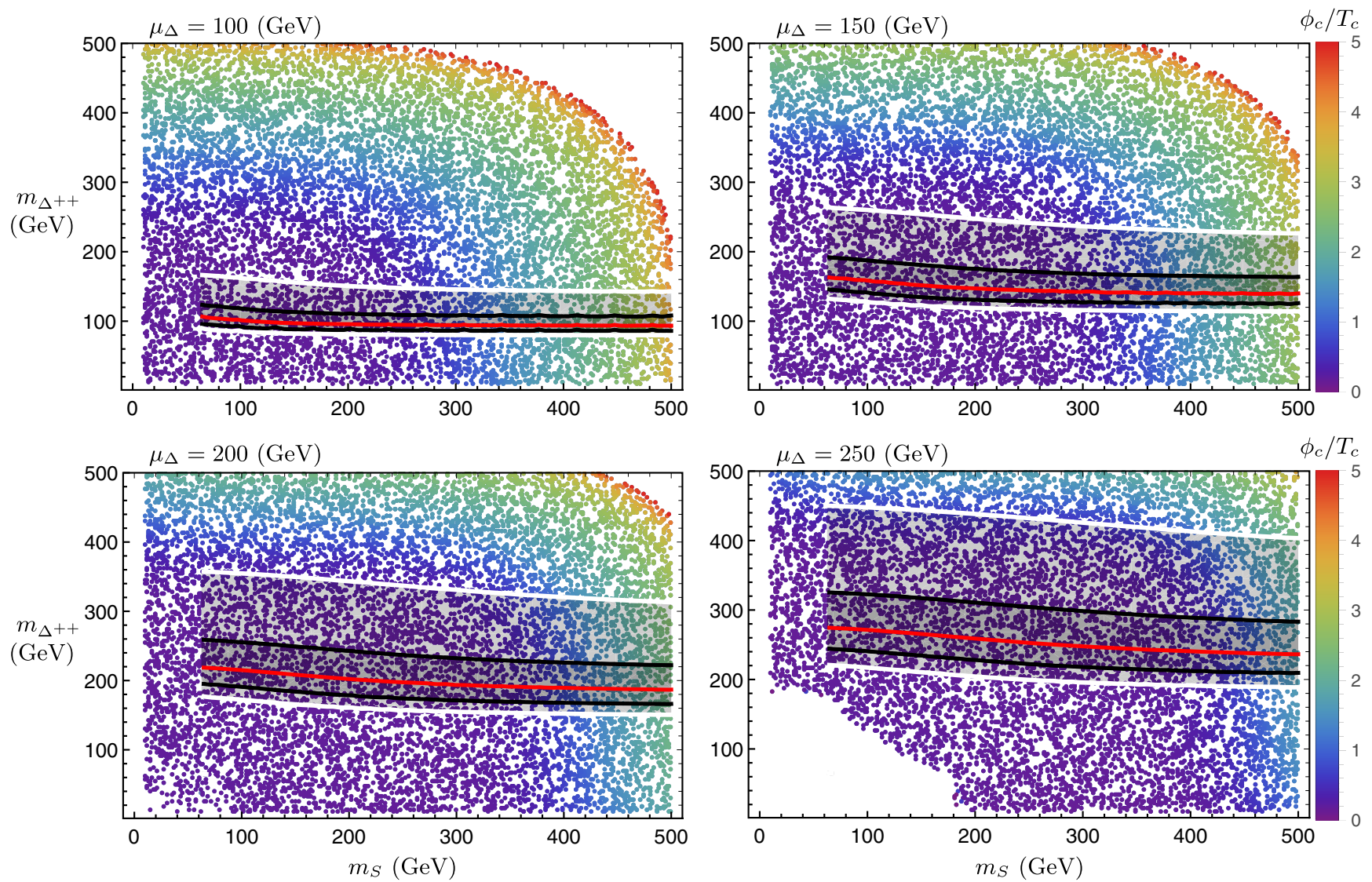

FIG. 4. Colored points represent the region with a first-order electroweak phase transition. The colors of the points demonstrate the strength of the phase transition, $\phi_{c} / T_{c}$, at the critical temperature. The red lines show parameter regions that are compatible with the ATLAS result for $R_{\gamma \gamma}$. The black (white) lines represent the boundary of the $1 \sigma(2 \sigma)$ bands for the ATLAS result.

regions with $\phi_{c} / T_{c}>1$ that are at the same time consistent with the experimental measurements of the diphoton Higgs boson decay rates.

\section{GRAVITATIONAL-WAVE SPECTRUM}

In this section we briefly review the processes for gravitational-wave $(\mathrm{GW})$ production following a first-order phase transition and then represent our result for the ITM extension of the SM.

There are three main sources of gravitational waves due to an electroweak phase transition: 1) collisions of the bubble walls and subsequent shocks in the plasma [43-49], 2) sound waves in the plasma generated after the bubbles have collided but before expansion has dissipated the kinetic energy in the plasma [50-53], and 3) magnetohydrodynamical (MHD) turbulence in the plasma formed after the bubbles have collided [54-58]. Generically, the three processes happen or coexist and thus the corresponding contributions to the gravitational waves' power spectrum, $\Omega h^{2}(f)$, must linearly combine at least approximately so that

$$
\Omega h^{2}(f)=\Omega_{\mathrm{col}} h^{2}(f)+\Omega_{\mathrm{sw}} h^{2}(f)+\Omega_{\mathrm{tur}} h^{2}(f),
$$

where $\Omega_{\mathrm{col}} h^{2}, \Omega_{\mathrm{sw}} h^{2}$, and $\Omega_{\mathrm{tur}} h^{2}$ represent the corresponding contribution from bubble collisions, sound waves, and turbulence, respectively. Now, let us briefly review each of these contributions in detail and estimate the predictions for the ITM.

Analytical studies and numerical simulations show that in order to estimate the gravitational-wave power spectrum due to a specific extension of the SM, one needs to supply at least three parameters $[59,60]$ : i) the ratio of released latent heat from the transition to the energy density of the plasma background, $\alpha$, ii) the time scale of the phase transition, $H_{*} / \beta$, and iii) the bubble wall velocity, $v_{b}$. Using the effective potential and its derivatives at the nucleation temperature $T_{n}$, the parameter $\alpha$ reads [59]

$\alpha=\left.\frac{1}{\rho_{R}}\left[-\left(V_{\mathrm{EW}}-V_{f}\right)+T_{n}\left(\frac{d V_{\mathrm{EW}}}{d T}-\frac{d V_{f}}{d T}\right)\right]\right|_{T=T_{n}}$,

where $V_{f}$ is the value of the potential in the unstable vacuum, $V_{\mathrm{EW}}$ is the value of the potential in the final vacuum, and $\rho_{R}$ is the energy density of the radiation bath, $\rho_{R}=g_{*} \pi^{2} T_{n}^{4} / 30$. The time scale of the phase transition can be calculated as [59] 


$$
\frac{H_{*}}{\beta}=\left.\left[T \frac{d}{d T}\left(\frac{S_{3}(T)}{T}\right)\right]^{-1}\right|_{T=T_{n}},
$$

where $S_{3}(T)$ is the three-dimensional Euclidean action of the critical bubble. The last ingredient from the phase transition is the velocity of the bubble wall $v_{b}$. The exact calculation of the bubble wall velocity is more complicated since one needs to consider bubble interactions with the background plasma. However, the bubble wall velocity can be estimated in terms of $\alpha$ as [61]

$$
v_{b}=\frac{1 / \sqrt{3}+\sqrt{\alpha^{2}+2 \alpha / 3}}{1+\alpha} .
$$

In fact, the above expression for the bubble wall velocity provides only a lower bound on the true wall velocity [48]. For some SM extensions, it has been checked that replacing the above approximation even with $v_{b}=1$, which is more appropriate for a very strong transition, does not significantly modify the results for gravitational-wave signals
[62]. For our analyses, we use the above approximation so that the parameters $\alpha$ and $\beta$ are sufficient in order to calculate the GW signals.

Now, based on the numerical simulations the peak frequency of GWs generated by bubble collisions [48], sound waves [52,53], and Kolmogorov-type turbulence [57] are, respectively, given by

$$
\begin{aligned}
f_{\text {sw }} & =1.9 \times 10^{-5} \frac{\beta}{H_{*}} \frac{1}{v_{b}} \frac{T_{n}}{100}\left(\frac{g_{*}}{100}\right)^{\frac{1}{6}} \mathrm{~Hz}, \\
f_{\text {turb }} & =2.7 \times 10^{-5} \frac{\beta}{H_{*}} \frac{1}{v_{b}} \frac{T_{n}}{100}\left(\frac{g_{*}}{100}\right)^{\frac{1}{6}} \mathrm{~Hz}, \\
f_{\text {col }} & =16.5 \times 10^{-6} \frac{0.62}{v_{b}^{2}-0.1 v_{b}+1.8} \frac{\beta}{H_{*}} \frac{T_{n}}{100}\left(\frac{g_{*}}{100}\right)^{\frac{1}{6}} \mathrm{~Hz} .
\end{aligned}
$$

Here $g_{*}$ is the number of relativistic degrees of freedom in the plasma at $T_{n}$. The energy densities of the GW spectrum, corresponding to each of the three contributions, are given by

$$
\begin{gathered}
\Omega h_{\mathrm{col}}^{2}(f)=1.67 \times 10^{-5}\left(\frac{\beta}{H_{*}}\right)^{-2} \frac{0.11 v_{b}^{3}}{0.42+v_{b}^{2}}\left(\frac{\kappa_{\mathrm{col}} \alpha}{1+\alpha}\right)^{2}\left(\frac{g_{*}}{100}\right)^{-\frac{1}{3}} \frac{3.8\left(f / f_{\mathrm{col}}\right)^{2.8}}{1+2.8\left(f / f_{\mathrm{col}}\right)^{3.8}}, \\
\Omega h_{\mathrm{sw}}^{2}(f)=2.65 \times 10^{-6}\left(\frac{\beta}{H_{*}}\right)^{-1}\left(\frac{\kappa_{\mathrm{sw}} \alpha}{1+\alpha}\right)^{2}\left(\frac{g_{*}}{100}\right)^{-\frac{1}{3}} v_{b}\left(\frac{f}{f_{\mathrm{sw}}}\right)^{3}\left(\frac{7}{4+3\left(f / f_{\mathrm{sw}}\right)^{2}}\right)^{7 / 2}, \\
\Omega h_{\mathrm{turb}}^{2}(f)=3.35 \times 10^{-4}\left(\frac{\beta}{H_{*}}\right)^{-1}\left(\frac{\kappa_{\mathrm{turb}} \alpha}{1+\alpha}\right)^{\frac{3}{2}}\left(\frac{g_{*}}{100}\right)^{-\frac{1}{3}} v_{b} \frac{\left(f / f_{\mathrm{turb}}\right)^{3}}{\left[1+\left(f / f_{\text {turb }}\right)\right]^{\frac{11}{3}}\left(1+8 \pi f / h_{*}\right)} .
\end{gathered}
$$

Here $h_{*}$ is the Hubble rate at the nucleation temperature, $h_{*}=16.5 \mu \mathrm{Hz}\left(\frac{T_{n}}{100 \mathrm{GeV}}\right)\left(g_{*} / 100\right)^{\frac{1}{6}}$, and $\kappa_{\text {cool }}, \kappa_{\text {sw }}$, and $\kappa_{\text {turb }}$ are efficiency factors.

The relative importance of each contribution to GW generation is encoded in the efficiency factors. These depend strongly on the dynamical details of the phase transition. In this regard, the velocity of the bubble wall plays a key role. Depending on the velocity of the bubble wall, there are two regimes: when the wall velocity is relativistic, and when it is not. Moreover, in the relativistic regime there are two different scenarios: when the bubble wall reaches a terminal velocity (nonrunaway scenario), and when the bubble wall accelerates without bound (runaway scenario). To calculate the GW spectrum, it is important to know which of the aforementioned scenarios apply. For this, the critical value $\alpha_{\infty}$ can be used to distinguish between these two scenarios $[59,63]$,

$$
\alpha_{\infty} \simeq \frac{30}{24 \pi^{2}} \frac{\sum_{a} c_{a} \Delta m_{a}^{2}}{g_{*} T_{n}^{2}} .
$$

Here, $c_{a}=n_{a} / 2\left(c_{a}=n_{a}\right), n_{a}$ is the number of degrees of freedom for boson (fermion) species, and $\Delta m_{a}^{2}$ is the squared-mass difference of particles between two phases at the nucleation temperature.

For nonrunaway scenarios, $\alpha<\alpha_{\infty}$, the bubble wall velocity $v_{b}$ remains subluminal and the available energy is transformed into fluid motion. So the dominant contributions to GWs come from sound waves and MHD turbulence, $h^{2} \Omega_{\mathrm{GW}} \simeq h^{2} \Omega_{\mathrm{sW}}+h^{2} \Omega_{\mathrm{turb}}$, with the efficiency factors given by [59]

$$
\begin{aligned}
\kappa_{\mathrm{col}} & \simeq 0, \\
\kappa_{\mathrm{sw}} & =(1-\epsilon) \kappa, \\
\kappa_{\mathrm{turb}} & =\epsilon \kappa .
\end{aligned}
$$

Here, $\epsilon \approx 0.05$ and $\kappa$, in the small- and large- $v_{b}$ limits, is approximately given by

$\kappa \simeq \begin{cases}\alpha(0.73+0.083 \sqrt{\alpha}+\alpha)^{-1}, & v_{b} \sim 1, \\ v_{b}^{6 / 5} 6.9 \alpha(1.36-0.037 \sqrt{\alpha}+\alpha)^{-1}, & v_{b} \lesssim 0.1 .\end{cases}$

The full expressions for $\kappa$ are given in Ref. [63].

For the runaway scenario, $\alpha>\alpha_{\infty}$, the excess vacuum energy density leads to bubble acceleration and $v_{b}$ is 
bounded only by the speed of light, $v_{b}=1$. In this case, all three GW sources contribute with efficiency factors

$$
\begin{aligned}
\kappa_{\mathrm{col}} & =1-\frac{\alpha_{\infty}}{\alpha}, \\
\kappa_{\mathrm{sw}} & =(1-\epsilon) \kappa, \\
\kappa_{\mathrm{turb}} & =\epsilon \kappa,
\end{aligned}
$$

where in this case $\kappa$ is given by $[62,63]$

$$
\kappa=\frac{\alpha_{\infty}}{\alpha}\left(\frac{\alpha_{\infty}}{0.73+0.083 \sqrt{\alpha_{\infty}}+\alpha_{\infty}}\right) .
$$

For a more accurate calculation of GW spectra, we consider two corrections which were discovered in recent studies [64,65]. First, we consider the correction of efficiency factors for strong transitions. The values of $\kappa$ given above are from a semianalytical hydrodynamic analysis. These are good estimations of $\kappa$ only for relatively weak transitions with $\alpha \ll 1$. For strong transitions and small $v_{b}$, a recent simulation found that $\kappa$ as specified in Eq. (21) gives an overestimation [64]. Using the numerical results in Ref. [64], we refine the estimation of the efficiency factor [66]. Second, we consider an additional suppression factor $\Upsilon$ in the $\Omega h_{\mathrm{sw}}^{2}$, which originates from the finite lifetime $\tau_{\mathrm{sw}}$ of the sound waves [65],

$$
\Upsilon=1-\frac{1}{\sqrt{1+2 \tau_{\mathrm{sw}} H_{*}}}
$$

For the classical approach, $\tau_{\mathrm{sw}} \rightarrow \infty$ is usually assumed which corresponds to the asymptotic $\Upsilon \rightarrow 1$. The lifetime $\tau_{\mathrm{sw}}$ can be considered as the time scale when the turbulence develops, approximately given by $[67,68]$

$$
\tau_{\mathrm{sw}} \sim \frac{R_{*}}{\bar{U}_{f}},
$$

where $R_{*}$ is the mean bubble separation and is related to $\beta$ through the relation $R_{*}=(8 \pi)^{1 / 3} v_{b} / \beta$ for an exponential bubble nucleation [69]. Further, the analysis performed in Ref. [69] was based on Minkowski spacetime. For an analysis based on an expanding universe, see Ref. [65]. The denominator $\bar{U}_{f}$ is the root-mean-squared fluid velocity which can be obtained from hydrodynamic analyses as $\bar{U}_{f}=\sqrt{\left(3 \kappa_{\nu} \alpha / 4\right)}[69,70]$. In Fig. 5, we explicitly show the effect of these corrections for our analyses for a typical point in the ITM parameter space.

Considering the above corrections, we show in Fig. 6 the $\mathrm{GW}$ power spectra for selected points with various $\phi_{c} / T_{c}$ values. We find that the peak frequencies and strengths of the gravitational-wave signals are strongly correlated with the strength of the phase transition.
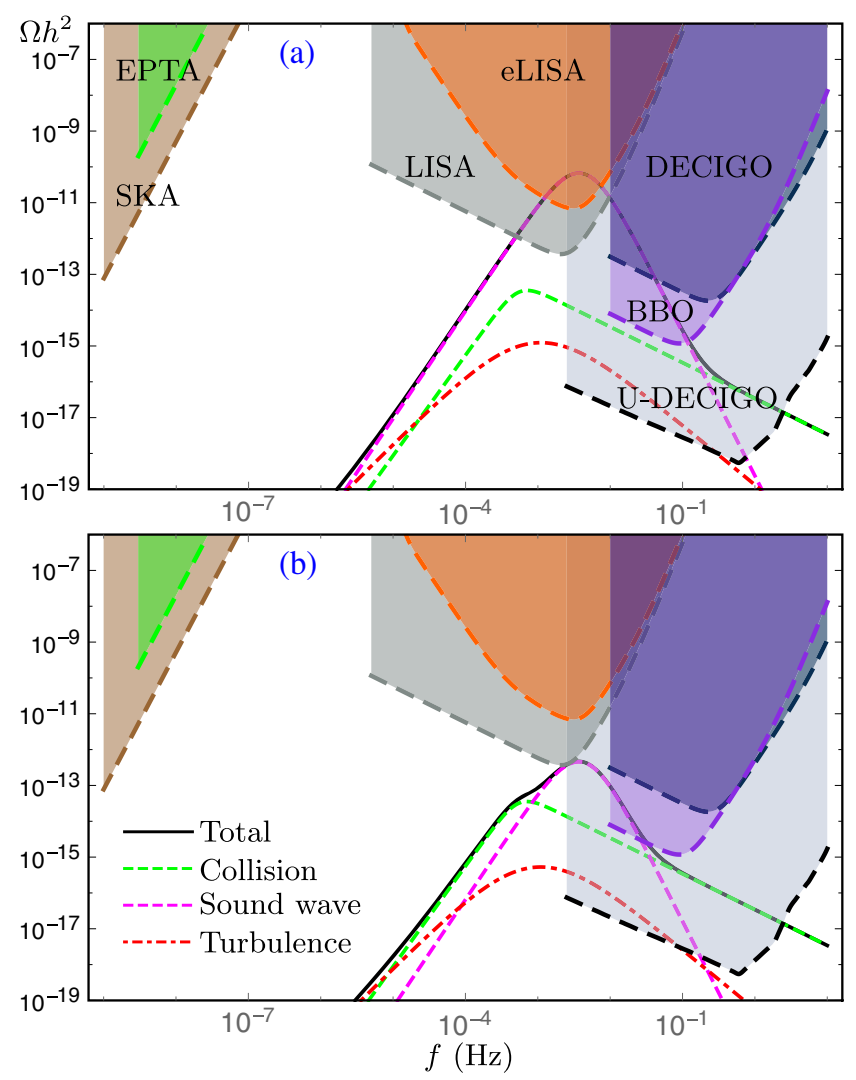

FIG. 5. Spectra of the GWs from the electroweak phase transition for a typical point in the parameter space; $m_{\Delta^{++}}=335.3 \mathrm{GeV}, m_{S}=426 \mathrm{GeV}$, and $\mu_{\Delta}=100 \mathrm{GeV}$. The shaded regions represent the expected sensitivities of $\mathrm{GW}$ interferometers. (a): the GW spectrum is computed using the semianalytical hydrodynamic approximation (14)-(16). (b): same as in panel (a) but with the corrected $\kappa$ parameter and the modification due to the finite lifetime of the sound waves.

Next, in order to assess the detectability of the GW signal by a given detector, one needs to consider the signal-tonoise ratio (SNR) over the running time of the detector $t_{\mathrm{obs}}$, which is given by $[23,71]$

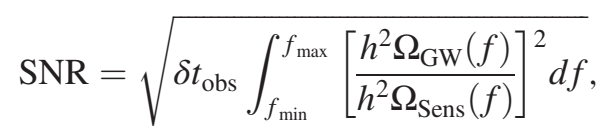

where $h^{2} \Omega_{\text {Sens }}(f)$ represents the sensitivity of the detector. The interval of integration $\left[f_{\min }, f_{\max }\right]$ is the frequency bandwidth of the detector. The factor $\delta$, which indicates the number of independent channels for the GW detector, is equal to 2 for $\mathrm{BBO}$ and U-DECIGO and equal to 1 for the rest. We consider $t_{\mathrm{obs}}=5$ years for all detectors. Whenever the SNR turns out to be larger than some threshold value, $\mathrm{SNR}>\mathrm{SNR}_{\mathrm{thr}}$, one can assert that the experiment under consideration will be able to detect the GW signal. The method of quantifying $\mathrm{SNR}_{\text {thr }}$ was briefly described in Ref. [23]. For example, the SNR threshold for discovery at eLISA is 10 or 50 , depending on the operating 

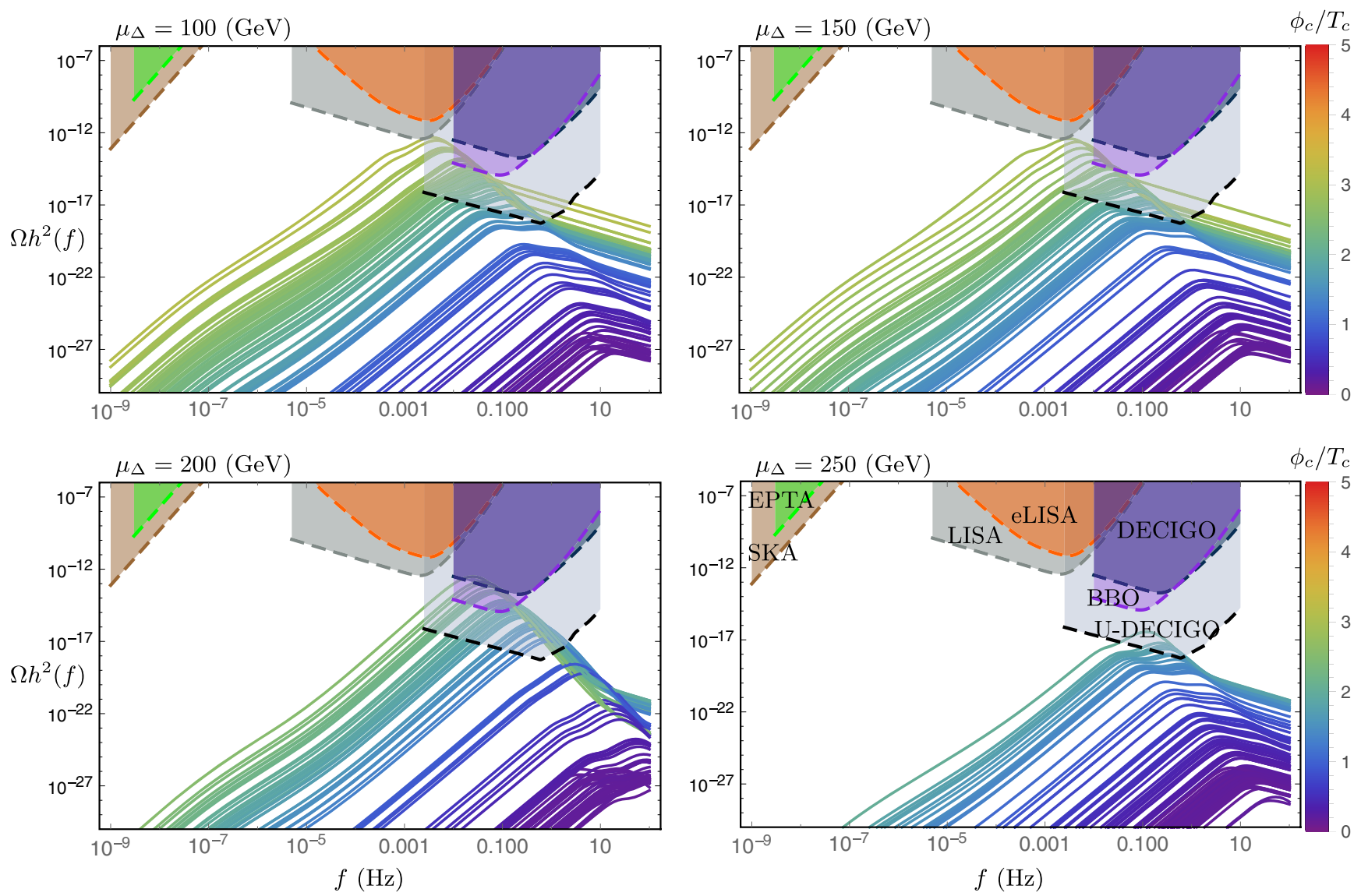

FIG. 6. Spectra of GWs from the electroweak phase transition for randomly sampled examples from the colored points in Fig. 4, i.e., the points with a strong first-order EWPT. The sensitivity regions for prospective GW detectors such as eLISA, BBO, and DECIGO are also shown. It can be seen that the intensity of the GW signal increases with the strength of the phase transition, i.e., $\phi_{c} / T_{c}$. For comparison, we also show the sensitivity regions for the SKA and EPTA detectors which cannot probe any part of the parameter space of the inert complex triplet model.

configuration [23]. Here we compute the signal-to-noise ratio for the eLISA, LISA, BBO, DECIGO, and U-DECIGO detectors. The results are shown in Fig. 7.

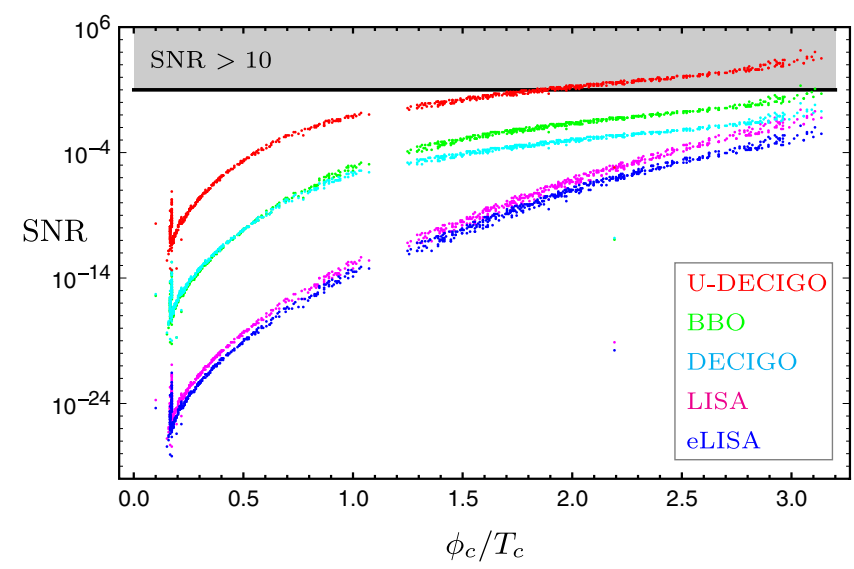

FIG. 7. Scatterplot of the signal-to-noise ratio versus $\phi_{c} / T_{c}$ for the ITM-predicted GW signals based on different gravitationalwave detectors.
Based on the results presented in Fig. 7, the computed SNRs of eLISA are less than its threshold, i.e., 10, which means that these gravitational waves are not detectable by eLISA. The biggest SNRs are associated with U-DECIGO. In fact, for points with $\phi_{c} / T_{c} \sim 3$, calculations concerning U-DECIGO lead to an SNR $\sim 100$. However, since the $\mathrm{SNR}_{\text {thr }}$ of U-DECIGO is not known (to the best of our knowledge), the question remains whether it can detect such GWs arising from an EWPT with $\phi_{c} / T_{c} \sim 3$.

\section{SUMMARY}

We have investigated the cosmological electroweak phase transition in an inert triplet scalar extension of the SM model. We found that there are regions of parameter space that can both yield a strong first-order electroweak phase transition and be consistent with recent LHC results on the Higgs-to-diphoton decay rate.

In principle, a first-order cosmological phase transition can lead to a background GW stochastic. Besides collider phenomenology, this can be used to probe the parameter space of particle physics models beyond the SM. In this 
regard, considering the recent treatment of GW spectrum estimation $[64,65]$, we studied the GW signals generated after the first-order electroweak phase transitions within the framework of the inert triplet model.

Based on the signal-to-noise ratio analyses, we found that very sensitive GW detectors will be needed to detect the inert triplet model signal. Probing the GW signals of this model will be difficult or maybe impossible for prospective space-based GW detectors with less sensitive configurations compared to U-DECIGO.

We also computed the $H \rightarrow Z \gamma$ decay rate in this model, which can be used as a probe at future collider experiments, such as the High-Luminosity LHC and other colliders with higher center-of-mass energies [33].

\section{ACKNOWLEDGMENTS}

We thank H. Hashamipour, A. Kargaran, and L. Kalhor for conversations, useful discussions, and comments while working on this project. We also sincerely thank the referee for their insightful comments towards the improvement of this paper.

\section{APPENDIX A: HIGGS DECAY WIDTHS}

For completeness, below we summarize the decay widths of the Higgs boson, which are the same in both the SM and ITM.

\section{Decay to leptons}

In the Born approximation, the partial decay width of $h$ to any fermion channel is $[26,72]$

$$
\Gamma_{h \rightarrow f \bar{f}}=\frac{N_{c} g^{2}}{32 \pi m_{W}^{2}} m_{h} m_{f}^{2}\left(1-\frac{4 m_{f}^{2}}{m_{h}^{2}}\right)^{3 / 2},
$$

where the color factor $N_{c}$ is 1 for leptons and 3 for quarks.

$$
\begin{aligned}
& \text { 2. Decay to quarks [73] } \\
& \Gamma(h \rightarrow q \bar{q})=\frac{N_{c} g^{2}}{32 \pi m_{W}^{2}} m_{h} \bar{m}_{q}^{2}\left(m_{h}\right)\left\{1+5.67 \frac{\bar{\alpha}_{s}\left(m_{h}\right)}{\pi}\right. \\
& +\left[37.51-1.36 N_{f}-\frac{2}{3} \log \frac{m_{h}^{2}}{m_{t}^{2}}\right. \\
& \left.\left.+\left(\frac{1}{3} \log \frac{\bar{m}_{q}^{2}\left(m_{h}\right)}{m_{h}^{2}}\right)^{2}\right] \frac{\bar{\alpha}_{s}^{2}\left(m_{h}\right)}{\pi^{2}}\right\},
\end{aligned}
$$

where, at the one-loop level, the running strong coupling constant is approximated as [74]

$$
\bar{\alpha}_{s}\left(m_{h}\right)=\frac{\bar{\alpha}_{s}\left(m_{Z}\right)}{1+\frac{33-2 N_{f}}{12 \pi} \bar{\alpha}_{s}\left(m_{Z}\right) \log \frac{m_{h}^{2}}{m_{Z}^{2}}},
$$

$N_{f}=5$, and the running quark mass defined at the scale $m_{h}$ is [75]

$$
\begin{aligned}
\bar{m}_{q}\left(m_{h}\right)= & \bar{m}_{q}\left(m_{q}\right)\left(\frac{\bar{\alpha}_{s}\left(m_{h}\right)}{\bar{\alpha}_{s}\left(m_{q}\right)}\right)^{12 /\left(33-2 N_{f}\right)} \\
& \times \frac{1+c_{1 q} \bar{\alpha}_{s}\left(m_{h}\right) / \pi+c_{2 q} \bar{\alpha}_{s}^{2}\left(m_{h}\right) / \pi^{2}}{1+c_{1 q} \bar{\alpha}_{s}\left(m_{q}\right) / \pi+c_{2 q} \bar{\alpha}_{s}^{2}\left(m_{q}\right) / \pi^{2}},
\end{aligned}
$$

in which $c_{1 b}=1.17, c_{2 b}=1.50$ and $c_{1 c}=1.01, c_{2 c}=1.39$ for the bottom and charm quark, respectively. The strong coupling and the quark masses are taken from Ref. [74]: $\bar{\alpha}_{s}\left(M_{Z}\right)=0.118, \bar{\alpha}_{s}\left(m_{b}\right)=0.223, \bar{\alpha}_{s}\left(m_{c}\right)=0.38, \bar{m}_{c}\left(m_{c}\right)=$ $1.273 \mathrm{GeV}$, and $\bar{m}_{b}\left(m_{b}\right)=4.18 \mathrm{GeV}$.

\section{Decay to gauge bosons}

The decay width of Higgs decay to gluons via quark loops is given by [26]

$$
\Gamma_{h \rightarrow g g}=\frac{\alpha_{s}^{2} g^{2} m_{h}^{3}}{128 \pi^{3} m_{W}^{2}}\left|\sum_{i} \frac{1}{2} A_{1 / 2}\left(\frac{4 m_{i}^{2}}{m_{h}^{2}}\right)\right|^{2}
$$

where the sum is over all quarks, $i=(t, b, c, s, u, d)$; however, the main contribution comes from the top quark. The loop function $A_{1 / 2}(x)$ is represented in Appendix B. Finally, the decay widths of $h \rightarrow Z Z^{*}$ and $h \rightarrow W W^{*}$, summed over all available channels, are given by $[26,76]$

$$
\Gamma_{h \rightarrow V V^{*}}=\frac{3 g^{4}}{512 \pi^{3}} m_{h} \delta_{V} R_{T}\left(\frac{m_{V}^{2}}{m_{h}^{2}}\right),
$$

where

$$
\begin{aligned}
R_{T}(x)= & \frac{3\left(1-8 x+20 x^{2}\right)}{\sqrt{4 x-1}} \arccos \left(\frac{3 x-1}{2 x^{3 / 2}}\right) \\
& -\frac{|1-x|}{2 x}\left(2-13 x+47 x^{2}\right) \\
& -\frac{3}{2}\left(1-6 x+4 x^{2}\right) \log x, \\
\text { and } \delta_{W}=1, \delta_{Z} & =\frac{1}{\cos ^{4} \theta_{W}}\left(\frac{7}{12}-\frac{10}{9} \sin ^{2} \theta_{W}+\frac{40}{27} \sin ^{4} \theta_{W}\right) .
\end{aligned}
$$

\section{APPENDIX B: LOOP FUNCTIONS}

The loop functions $A_{(0,1 / 2,1)}^{\gamma \gamma}$ and $A_{(0,1 / 2,1)}^{Z \gamma}$ are defined as follows $[26,77]$ : 


$$
\begin{aligned}
A_{0}^{\gamma \gamma}(x) & =-x^{2}\left[x^{-1}-f(x)\right], \\
A_{1 / 2}^{\gamma \gamma}(x) & =2 x^{2}\left[x^{-1}+\left(x^{-1}-1\right) f(x)\right], \\
A_{1}^{\gamma \gamma}(x) & =-x^{2}\left[2 x^{-2}+3 x^{-1}+3\left(2 x^{-1}-1\right) f(x)\right], \\
A_{0}^{Z \gamma}(x, y) & =I_{1}(x, y), \\
A_{1 / 2}^{Z \gamma}(x, y) & =I_{1}(x, y)-I_{2}(x, y), \\
A_{1}^{Z \gamma}(x, y) & =4\left(3-\tan ^{2} \theta_{W}\right) I_{2}(x, y)+\left[\left(1+2 x^{-1}\right) \tan ^{2} \theta_{W}-\left(5+2 x^{-1}\right)\right] I_{1}(x, y),
\end{aligned}
$$

where

$$
\begin{aligned}
& I_{1}(x, y)=\frac{x y}{2(x-y)}+\frac{x^{2} y^{2}}{2(x-y)^{2}}[f(x)-f(y)]+\frac{x^{2} y}{(x-y)^{2}}[g(x)-g(y)], \\
& I_{2}(x, y)=-\frac{x y}{2(x-y)}[f(x)-f(y)],
\end{aligned}
$$

where the functions $f(x)$ and $g(x)$ are given by

$$
\begin{gathered}
f(\tau)= \begin{cases}\arcsin ^{2}\left(\frac{1}{\sqrt{\tau}}\right) & \text { for } \tau \geq 1, \\
-\frac{1}{4}\left[\log \left(\frac{1+\sqrt{1-\tau}}{1-\sqrt{1-\tau}}\right)-i \pi\right]^{2} & \text { for } \tau<1,\end{cases} \\
g(\tau)= \begin{cases}\sqrt{\tau-1} \arcsin (1 / \sqrt{\tau}) & \text { for } \tau \geq 1, \\
\frac{1}{2} \sqrt{1-\tau}\left(\log \frac{1+\sqrt{1-\tau}}{1-\sqrt{1-\tau}}-i \pi\right) & \text { for } \tau<1 .\end{cases}
\end{gathered}
$$

[1] T. Vachaspati, Magnetic fields from cosmological phase transitions, Phys. Lett. B 265, 258 (1991); A. Brandenburg, K. Enqvist, and P. Olesen, Large-scale magnetic fields from hydromagnetic turbulence in the very early universe, Phys. Rev. D 54, 1291 (1996); M. Joyce and M. Shaposhnikov, Primordial Magnetic Fields, Right Electrons, and the Abelian Anomaly, Phys. Rev. Lett. 79, 1193 (1997); D. Boyanovsky, H. J. de Vega, and M. Simionato, Magnetic field generation from nonequilibrium phase transitions, Phys. Rev. D 67, 023502 (2003); T. Stevens, M. B. Johnson, L. S. Kisslinger, E. M. Henley, W.-Y.P. Hwang, and M. Burkardt, Role of charged gauge fields in generating magnetic seed fields in bubble collisions during the cosmological electroweak phase transition, Phys. Rev. D 77, 023501 (2008); C. J. Copi, F. Ferrer, T. Vachaspati, and A. Achúcarro, Helical Magnetic Fields from Sphaleron Decay and Baryogenesis, Phys. Rev. Lett. 101, 171302 (2008); T. Kahniashvili, A. G. Tevzadze, A. Brandenburg, and A. Neronov, Evolution of primordial magnetic fields from phase transitions, Phys. Rev. D 87, 083007 (2013); A. Díaz-Gil, J. García-Bellido, M. G. Pérez, and A. González-Arroyo,
Magnetic Field Production During Preheating at the Electroweak Scale, Phys. Rev. Lett. 100, 241301 (2013); Y. Zhang, F. Ferrer, and T. Vachaspati, Vacuum topology and the electroweak phase transition, Phys. Rev. D 96, 043014 (2017); Y. Zhang, T. Vachaspati, and F. Ferrer, Magnetic field production at a first-order electroweak phase transition, Phys. Rev. D 100, 083006 (2019).

[2] M. Hindmarsh, S. J. Huber, K. Rummukainen, and D. J. Weir, Gravitational Waves from the Sound of a First Order Phase Transition, Phys. Rev. Lett. 112, 041301 (2014); M. Kakizaki, S. Kanemura, and T. Matsui, Gravitational waves as a probe of extended scalar sectors with the first order electroweak phase transition, Phys. Rev. D 92, 115007 (2015); D. J. Weir, Gravitational waves from a first-order electroweak phase transition: A brief review, Phil. Trans. R. Soc. A 376, 20170126 (2018).

[3] C. Wainwright and S. Profumo, Impact of a strongly firstorder phase transition on the abundance of thermal relics, Phys. Rev. D 80, 103517 (2009).

[4] M. Trodden, Electroweak baryogenesis, Rev. Mod. Phys. 71, 1463 (1999). 
[5] P. A. R. Ade et al. (Planck Collaboration), Planck 2015 results. XIII. Cosmological parameters, Astron. Astrophys. 594, A13 (2016).

[6] R. H. Cyburt, B. D. Fields, K. A. Olive, and T-H. Yeh, Big bang nucleosynthesis: Present status, Rev. Mod. Phys. 88, 015004 (2016).

[7] G. Steigman, Annu. Rev. Astron. Astrophys. 14, 339 (1976);

A. G. Cohen, A. De Rjula, and S. L. Glashow, A matterantimatter universe?, Astrophys. J. 495, 539 (1998); C. Bambi and A.D. Dolgov, Antimatter in the Milky Way, Nucl. Phys. B784, 132 (2007); A. G. Mayorov, A. M. Galper, O. Adriani et al., Upper limit on the antihelium flux in primary cosmic rays, JETP Lett. 93, 628 (2011); D. A. Prokhorov, Upper bounds on matter-antimatter admixture from gamma-ray observations of colliding clusters of galaxies with the Fermi large area telescope, Phys. Rev. D 91, 083002 (2015).

[8] A. D. Sakharov, Violation of $C P$ invariance, C asymmetry, and baryon asymmetry of the universe, Zh. Eksp. Teor. Fiz. Pisma 5, 32 (1967) [Sov. Phys. Usp. 34, 362 (1991)].

[9] K. Kajantie, M. Laine, K. Rummukainen, and M. Shaposhnikov, Is There a Hot Electroweak Phase Transition at $m_{H} \gtrsim m_{W}$ ?, Phys. Rev. Lett. 77, 2887 (1996); F. Csikor, Z. Fodor, and J. Heitger, End Point of the Hot Electroweak Phase Transition, Phys. Rev. Lett. 82, 21 (1999); M. D’Onofrio, K. Rummukainen, and A. Tranberg, Sphaleron Rate in the Minimal Standard Model, Phys. Rev. Lett. 113, 141602 (2014); M. D'Onofrio and K. Rummukainen, Standard model cross-over on the lattice, Phys. Rev. D 93, 025003 (2016).

[10] M. Cirelli, N. Fornengo, and A. Strumia, Minimal dark matter, Nucl. Phys. B753, 178 (2006).

[11] S. S. AbdusSalam and T. A. Chowdhury, Scalar representations in the light of electroweak phase transition and cold dark matter phenomenology, J. Cosmol. Astropart. Phys. 05 (2014) 026.

[12] D. Curtin, P. Meade, and C. Yu, Testing electroweak baryogenesis with future colliders, J. High Energy Phys. 11 (2014) 127; A. Beniwal, M. Lewicki, J. D. Wells, M. White, and A. G. Williams, Gravitational wave, collider and dark matter signals from a scalar singlet electroweak baryogenesis, J. High Energy Phys. 08 (2017) 108; G. Kurup and M. Perelstein, Dynamics of electroweak phase transition in singlet-scalar extension of the standard model, Phys. Rev. D 96, 015036 (2017); V. Vaskonen, Electroweak baryogenesis and gravitational waves from a real scalar singlet, Phys. Rev. D 95, 123515 (2017).

[13] T. Alanne, T. Hugle, M. Platscher, and K. Schmitz, A fresh look at the gravitational-wave signal from cosmological phase transitions, J. High Energy Phys. 03 (2020) 004.

[14] T. G. Steele, Z. W. Wang, D. Contreras, and R. B. Mann, Viable Dark Matter via Radiative Symmetry Breaking in a Scalar Singlet Higgs Portal Extension of the Standard Model, Phys. Rev. Lett. 112, 171602 (2014).

[15] S. Baum, M. Carena, N. R. Shah, C. E. M. Wagner, and Y. Wang, Nucleation is more than critical—a case study of the electroweak phase transition in the NMSSM, J. High Energy Phys. 03 (2021) 055.

[16] T. A. Chowdhury, M. Nemevek, G. Senjanovi, and Y. Zhang, Dark matter as the trigger of strong electroweak phase transition, J. Cosmol. Astrophys. Phys. 02 (2012) 029; D. Borah and J. M. Cline, Inert doublet dark matter with strong electroweak phase transition, Phys. Rev. D 86, 055001 (2012); N. Blinov, J. Kozaczuk, D. E. Morrissey, and C. Tamarit, Electroweak baryogenesis from exotic electroweak symmetry breaking, Phys. Rev. D 92, 035012 (2015); N. Blinov, S. Profumo, and T. Stefaniak, The electroweak phase transition in the inert doublet model, J. Cosmol. Astropart. Phys. 07 (2015) 028; A. Paul, B. Banerjee, and D. Majumdar, Gravitational wave signatures from an extended inert doublet dark matter model, J. Cosmol. Astropart. Phys. 10 (2019) 062.

[17] F. Peng Huang and J.-H. Yu, Exploring inert dark matter blind spots with gravitational wave signatures, Phys. Rev. D 98, 095022 (2018).

[18] H.H. Patel and M. J. Ramsey-Musolf, Stepping into electroweak symmetry breaking: Phase transitions and Higgs phenomenology, Phys. Rev. D 88, 035013 (2013); M. Chala, M. Ramos, and M. Spannowsky, Gravitational wave and collider probes of a triplet Higgs sector with a low cutoff, Eur. Phys. J. C 79, 156 (2019).

[19] S. Abdussalam, M. J. Kazemi, and L. Kalhor, Upper limit on first-order electroweak phase transition strength, Int. J. Mod. Phys. A 36, 2150024 (2021).

[20] M. Magg and C. Wetterich, Neutrino mass problem and gauge hierarchy, Phys. Lett. 94B, 61 (1980); G. Lazarides, Q. Shafi, and C. Wetterich, Proton lifetime and fermion masses in an SO(10) model, Nucl. Phys. B181, 287 (1981); T. P. Cheng and L.-F. Li, Neutrino masses, mixings and oscillations in SU(2) U(1) models of electroweak interactions, Phys. Rev. D 22, 2860 (1980); R. N. Mohapatra and G. Senjanovic, Neutrino masses and mixings in gauge models with spontaneous parity violation, Phys. Rev. D 23, 165 (1981).

[21] J. Schechter and J. W. F. Valle, Neutrino masses in $\mathrm{SU}(2) \times$ U(1) theories, Phys. Rev. D 22, 2227 (1980).

[22] J. Schechter and J. W. F. Valle, Neutrino decay and spontaneous violation of lepton number, Phys. Rev. D 25, 774 (1982).

[23] C. Caprini, M. Hindmarsh, S. Huber, T. Konstandin, J. Kozaczuk, G. Nardini, J. Miguel No, A. Petiteau, P. Schwaller, G. Servant, and D. J. Weir, Science with the space-based interferometer eLISA. II: Gravitational waves from cosmological phase transitions, J. Cosmol. Astropart. Phys. 04 (2016) 001.

[24] T. Hambye, F.-S. Ling, L. Lopez Honorez, and J. Rocher, Scalar multiplet dark matter, J. High Energy Phys. 05 (2010) 066.

[25] A. Arhrib, R. Benbrik, and N. Gaur, $H \rightarrow \gamma \gamma$ in the inert Higgs doublet model, Phys. Rev. D 85, 095021 (2012).

[26] J. F. Gunion, H. E. Haber, G. L. Kane, and S. Dawson, The Higgs Hunter's guide, Front. Phys. 80, 1 (2000), https:// inspirehep.net/literature/279039.

[27] B. Świeżewska and M. Krawczyk, Diphoton rate in the inert doublet model with a $125 \mathrm{GeV}$ Higgs boson, Phys. Rev. D 88, 035019 (2013).

[28] C.-S. Chen, C.-Q. Geng, D. Huang, and L.-H. Tsai, New scalar contributions to $h \rightarrow Z \gamma$, Phys. Rev. D 87, 075019 (2013). 
[29] A. Denner, Techniques for the calculation of electroweak radiative corrections at the one-loop level and results for W-physics at LEP200, Fortschr. Phys. 41, 307 (1993).

[30] C.-S. Chen, C.-Q. Geng, D. Huang, and L.-H. Tsai, Correlation of $h \rightarrow \gamma \gamma$ and $Z \gamma$ in Type-II seesaw neutrino model, Phys. Lett. B 723, 156 (2013).

[31] ATLAS Collaboration, Measurements of Higgs boson properties in the diphoton decay channel with $36 \mathrm{fb} 1$ of pp collision data at $s=13 \mathrm{TeV}$ with the ATLAS detector, Phys. Rev. D 98, 052005 (2018).

[32] CMS Collaboration, Measurements of Higgs boson properties in the diphoton decay channel in proton-proton collisions at $s=13 \mathrm{TeV}$, J. High Energy Phys. 11 (2018) 185.

[33] F. Goertz, E. Madge, P. Schwaller, and V. T. Tenorth, Discovering the $h \rightarrow Z \gamma$ decay in $t \bar{t}$ associated production, Phys. Rev. D 102, 053004 (2020).

[34] ATLAS Collaboration, A search for the $Z \gamma$ decay mode of the Higgs boson in pp collisions at $\sqrt{s}=13 \mathrm{TeV}$ with the ATLAS detector, Phys. Lett. B 809, 135754 (2020).

[35] M. Quiros, Finite temperature field theory and phase transitions, arXiv:hep-ph/9901312.

[36] D. Croon, O. Gould, P. Schicho, T. V. I. Tenkanen, and G. White, Theoretical uncertainties for cosmological first-order phase transitions, arXiv:2009.10080.

[37] In the on-shell renormalization scheme with cutoff regularization.

[38] S. R. Coleman and E. J. Weinberg, Radiative corrections as the origin of spontaneous symmetry breaking, Phys. Rev. D 7, 1888 (1973).

[39] R. R. Parwani, Resummation in a hot scalar field theory, Phys. Rev. D 45, 4695 (1992).

[40] E. J. Weinberg and A.-q. Wu, Understanding complex perturbative effective potentials, Phys. Rev. D 36, 2474 (1987);

[41] J. M. Cline, 2018 Is electroweak baryogenesis dead?, Phil. Trans. Roy. Soc. Lond. A 376, 20170116 (2018).

[42] G. D. Moore, Measuring the broken phase sphaleron rate nonperturbatively, Phys. Rev. D 59, 014503 (1998).

[43] A. Kosowsky, M. S. Turner, and R. Watkins, Gravitational radiation from colliding vacuum bubbles, Phys. Rev. D 45, 4514 (1992).

[44] A. Kosowsky, M. S. Turner, and R. Watkins, Gravitational Waves from First Order Cosmological Phase Transitions, Phys. Rev. Lett. 69, 2026 (1992).

[45] A. Kosowsky and M. S. Turner, Gravitational radiation from colliding vacuum bubbles: Envelope approximation to many bubble collisions, Phys. Rev. D 47, 4372 (1993).

[46] M. Kamionkowski, A. Kosowsky, and M. S. Turner, Gravitational radiation from first order phase transitions, Phys. Rev. D 49, 2837 (1994).

[47] C. Caprini, R. Durrer, and G. Servant, Gravitational wave generation from bubble collisions in first-order phase transitions: An analytic approach, Phys. Rev. D 77, 124015 (2008).

[48] S. J. Huber and T. Konstandin, Gravitational wave production by collisions: More bubbles, J. Cosmol. Astropart. Phys. 09 (2008) 022.
[49] R. Jinno and M. Takimoto, Gravitational waves from bubble collisions: An analytic derivation, Phys. Rev. D 95, 024009 (2017).

[50] J. T. Giblin, Jr. and J. B. Mertens, Vacuum bubbles in the presence of a relativistic fluid, J. High Energy Phys. 12 (2013) 042.

[51] J. T. Giblin and J. B. Mertens, Gravitional radiation from first-order phase transitions in the presence of a fluid, Phys. Rev. D 90, 023532 (2014).

[52] M. Hindmarsh, S. J. Huber, K. Rummukainen, and D. J. Weir, Gravitational Waves from the Sound of a First Order Phase Transition, Phys. Rev. Lett. 112, 041301 (2014).

[53] M. Hindmarsh, S. J. Huber, K. Rummukainen, and D. J. Weir, Numerical simulations of acoustically generated gravitational waves at a first order phase transition, Phys. Rev. D 92, 123009 (2015).

[54] C. Caprini and R. Durrer, Gravitational waves from stochastic relativistic sources: Primordial turbulence and magnetic fields, Phys. Rev. D 74, 063521 (2006).

[55] T. Kahniashvili, A. Kosowsky, G. Gogoberidze, and Y. Maravin, Detectability of gravitational waves from phase transitions, Phys. Rev. D 78, 043003 (2008).

[56] T. Kahniashvili, L. Kisslinger, and T. Stevens, Gravitational radiation generated by magnetic fields in cosmological phase transitions, Phys. Rev. D 81, 023004 (2010).

[57] C. Caprini, R. Durrer, and G. Servant, The stochastic gravitational wave background from turbulence and magnetic fields generated by a first-order phase transition, J. Cosmol. Astropart. Phys. 12 (2009) 024.

[58] L. Kisslinger and T. Kahniashvili, Polarized gravitational waves from cosmological phase transitions, Phys. Rev. D 92, 043006 (2015).

[59] C. Caprini, M. Hindmarsh, S. Huber, T. Konstandin, J. Kozaczuk, G. Nardini, J. Miguel No, A. Petiteau, P. Schwaller, G. Servant, and D. J. Weir, Science with the space-based interferometer eLISA. II: Gravitational waves from cosmological phase transitions, J. Cosmol. Astropart. Phys. 04 (2016) 001.

[60] C. Grojean and G. Servant, Gravitational waves from phase transitions at the electroweak scale and beyond, Phys. Rev. D 75, 043507 (2007).

[61] P. J. Steinhardt, Relativistic detonation waves and bubble growth in false vacuum decay, Phys. Rev. D 25, 2074 (1982).

[62] A. Beniwal, M. Lewicki, J. D. Wells, M. White, and A. G. Williams, Gravitational wave, collider and dark matter signals from a scalar singlet electroweak baryogenesis, J. High Energy Phys. 08 (2017) 108.

[63] J. R. Espinosa, T. Konstandin, J. M. No, and G. Servant, Energy budget of cosmological first-order phase transitions, J. Cosmol. Astropart. Phys. 06 (2010) 028.

[64] D. Cutting, M. Hindmarsh, and D. J. Weir, Vorticity, Kinetic Energy, and Suppressed Gravitational Wave Production in Strong rst Order Phase Transitions, Phys. Rev. Lett. 125, 021302 (2020).

[65] H.-K. Guo, K. Sinha, D. Vagie, and G. White, Phase transitions in an expanding universe: Stochastic gravitational 
waves in standard and non-standard histories, J. Cosmol. Astropart. Phys. 01 (2021) 001.

[66] To this aim, we use of an interpolation of the numerical results which are represented in Fig. 3 of the Ref. [61].

[67] U.-L. Pen and N. Turok, Shocks in the Early Universe, Phys. Rev. Lett. 117, 131301 (2016).

[68] M. Hindmarsh, S. J. Huber, K. Rummukainen, and D. J. Weir, Shape of the acoustic gravitational wave power spectrum from a first order phase transition, Phys. Rev. D 96, 103520 (2017).

[69] M. Hindmarsh and M. Hijazi, Gravitational waves from first order cosmological phase transitions in the Sound Shell Model, J. Cosmol. Astropart. Phys. 12 (2019) 062.

[70] D. Weir, Gravitational waves from a first-order electro weak phase transition: A brief review, Phil. Trans. Roy. Soc. Lond. A 376, 20170126 (2018).
[71] K. Schmitz, New sensitivity curves for gravitational-wave signals from cosmological phase transitions, J. High Energy Phys. 01 (2021) 097.

[72] T. G. Rizzo, Decays of heavy Higgs bosons, Phys. Rev. D 22, 722 (1980).

[73] A. Djouadi, The anatomy of electroweak symmetry breaking: Tome I: The Higgs boson in the Standard Model, Phys. Rep. 457, 1 (2008).

[74] J. Beringer et al., Review of particle physics, Phys. Rev. D 86, 010001 (2012).

[75] A. Djouadi, J. Kalinowski, and P. Zerwas, Two-and threebody decay modes of SUSY Higgs particles, Z. Phys. C 70, 435 (1996).

[76] W.-Y. Keung and W. J. Marciano, Higgs-scalar decays: $H \rightarrow W^{ \pm}+X$, Phys. Rev. D 30, 248 (1984).

[77] M. Spira, QCD effects in Higgs physics, Fortschr. Phys. 46, 203 (1998). 